English, M. P. (1953). J. gen. Microbiol. 9, 15-25.

\title{
The Fermentation of Malt Extract by an Osmophilic Yeast
}

\author{
By MARY P. ENGLISH \\ Bacteriological Laboratory, Physiological Department, The British \\ Drug Houses, Ltd., Graham Street, London, N. 1 \\ With a Note by J. T. Gunner and Constance Rainbow on \\ a Method of Vital Staining for Saccharomyces rouxii
}

SUMMARY: The fermentation of malt extract by the osmophilic yeast Saccharomyces rouxii Boutroux is described. It is found that the limiting factor determining whether an extract will ferment or not is its relative humidity, the critical level of which is about $73 \%$. If the relative humidity is raised above this level, either by raising the moisture content or by lowering the proportion of sugars in the total solids, the extract can be fermented by $S$. rouxii.

This study of the biology of an osmophilic yeast began with the discovery of a malt extract and oil mixture which was fermenting vigorously. As this substance had a very low moisture content and contained a very high percentage of sugars, it was considered of interest to investigate the habit of any organism that could exploit conditions apparently so unpromising.

A preliminary investigation, of which a brief report has already appeared (English, 1951) showed that the chief causative organism of the fermentation was the yeast Zygosaccharomyces japonicus Saito now known as Saccharomyces rouxii Boutroux (Lodder \& Kreger-van Rij, 1952), and it is this organism only with which the present work is concerned.

Though there are records in the literature of the fermentation of a large number of high-sugar substrates by osmophilic yeasts (Mrak \& Phaff, 1948; von Schelhorn, 1951) no mention has so far been found of a fermentation of malt extract.

Just as there are organisms that can tolerate high sugar concentrations, so there are others which can survive in highly concentrated salt solutions and in substrates containing very low percentages of moisture. Stille (1948) found that dried foods were liable to fungal attack unless their relative humidity was less than $75 \%$, and it is known (Joslyn \& Creuss, 1929) that some yeasts can grow in pickle brine with a salt concentration of $19-20 \%$.

The yeast named $S$. rouxii was originally found in fermenting jams and syrups, and in solid glucose, by Boutroux (1884), and in recent years has been identified by Mossel (1951) as the cause of a fermentation of sugared fruits. On the other hand, it was in strong brine, not a high sugar substrate, that the organism first given the name of Z. japonicus was found by Saito (1907) while he was investigating the microbiology of the products of fermenting soya in the preparation of 'koji'. Z . japonicus has also been recorded, with other yeasts, by Fabian \& Quinet (1928) in honey, and by Fabian \& Hall (1933) in 
maple syrup. Also Mrak, Phaff \& Vaughn (1942) found that Z. japonicus variety soya was the dominant yeast in the flora of Egyptian and Californian dates.

Little is known of the effect of environment on Z. japonicus, but Fabian \& Quinet and Fabian \& Hall determined the moisture contents of fermented and unfermented samples of honey and maple syrups, and attempted to correlate these with yeast growth. Mrak et al. found that of all the yeasts they isolated from dates $Z$. japonicus was equalled only by two other Zygosaccharomyces species in its ability to grow in date syrup of $66^{\circ}$ Balling. Mossel gives the composition and concentration of the fruits on which he found S. rouxii.

The work described here is a study of the effect of a number of environmental factors on the growth of $S$. rouxii in malt extract.

\section{Description of the fermentation}

It is extremely difficult to say when fermentation of a malt extract actually begins: probably the first sign is the typical sickly-sweet, slightly 'beery' smell, reminiscent of that of fermenting jam. At the same time as this smell becomes noticeable, or perhaps slightly later, individual bubbles of gas appear throughout the extract and rise extremely slowly to the surface. If the malt remains undisturbed, this stage lasts a minimum of 3-4 weeks, often considerably longer, the bubbles accumulating very slowly at the surface. At a certain point, however, the malt extract appears to become saturated with the gas, numerous minute bubbles form comparatively quickly throughout the mass, and within 10-14 days a thick layer of golden-yellow froth forms over the surface of the malt and may begin to ooze from the container. This comparatively sudden frothing can be induced quite early in the fermentation by simply stirring the malt slightly, when the same thick froth will form in 2 or 3 days, indicating that the malt becomes supersaturated with gas which can be liberated, either spontaneously when the limit of saturation is reached, or by mechanical disturbance before this point. The same phenomenon can be observed when a pure culture of the yeast is grown in a strong glucose solution. This may be stored for several days with no visible fermentation, but if it is then shaken, a mass of bubbles forms throughout the tubes some seconds, or even minutes, later.

The consistency of malt extract is such that when active fermentation is over and the yeasts are decreasing in numbers, the gas bubbles are still held by the malt, which may retain the appearance of being in a state of active fermentation for several months.

\section{MA'TERIALS AND METHODS}

The original fermentation was found in a malt extract and oil mixture : similar mixtures were therefore used as substrates in this work. Mixture A came from the same source as that in which the fermentation was first found, and contained $5 \%(w / w)$ of oil, while mixture $B$ contained an extract of different composition and $10 \%(w / w)$ of oil. Mixture A was used except where $\mathbf{B}$ is 
specified. There was some variation of composition from batch to batch of both extracts, and in particular the moisture content of mixture $\mathbf{A}$ varied between 19.4 and $21.5 \%$, while that of mixture $B$ was between 19.3 and $20.6 \%$.

Complete sterilization of malt extract for experimental purposes is impracticable, as the high temperatures necessary cause changes in its colour and composition. A technique of 'partial sterilization' was therefore adopted, where the extract was held at $63^{\circ}$ for $1 \mathrm{hr}$. in a water-bath, a procedure which left the extract unchanged while killing off any fermentative organisms. Fermentation never occurred in uninoculated malt treated thus.

Small-scale experiments with malt extract were carried out in $6 \times 1$ in. boiling tubes, the plugs of which were covered with rubber caps to prevent excessive evaporation. For larger scale work, sterile, $450 \mathrm{ml}$. clear-glass screw-cap bottles, effectively sealed against evaporation, were used. Enough inoculum for a whole experiment was prepared by suspending yeast cells, scraped from slope cultures or centrifuged from liquid cultures, in the minimum quantity of concentrated maltose solution (so as to dilute the malt extract as little as possible) and mixing it thoroughly in about $\frac{1}{2} \mathrm{lb}$. of yeast-free malt extract. This in turn was used to inoculate the main bulk of partially sterilized malt extract contained in a large, sterile earthenware jar, the prepared glass jars being then half-filled with the inoculated malt, sealed and incubated. All manipulations involving exposure of the extract to the air were carried out in a sterile room. Fermentation was considered to have begun when bubbles could be seen, and the typical 'fermented' smell was apparent, but it must be emphasized that, in practice, it was impossible to tell the exact time of the onset of fermentation, and times given in the results of experiments refer to stages where there was no doubt that it had started. In unstirred samples the lag period may run into weeks.

The number of yeasts in any given sample of malt extract was estimated by a modified dilution plate technique. As it had been found that temporary suspension in distilled water did not affect the viability of the yeast cells, the extract was weighed into distilled water to give a dilution of $1 / 10$, dissolved by shaking, and $1 \mathrm{ml}$. of the solution, or of known dilutions of it, was mixed with about $7 \mathrm{ml}$. portions of an agar medium and plates poured, the colonies being counted after 4 days' incubation at $30^{\circ}$. The agar medium used was originally adapted from the selective medium of de Whalley \& Scarr (1947) by the substitution of glucose for sucrose, but later this was found to be unnecessarily rich for $S$. rouxii, the following simplified formula proving quite satisfactory: $15 \mathrm{~g}$. powdered malt extract, $0.78 \mathrm{~g}$. peptone, $450 \mathrm{~g}$. glucose, $15 \mathrm{~g}$. powdered agar, 11 . distilled water.

As the yeast colonies on this medium were small and difficult to count, a method of vital staining with Trypan Blue, suggested and worked out by Mr J. T. Gunner and Miss Constance Rainbow of this laboratory, was adopted as a routine. The method is described in a note at the end of this paper.

When $S$. rouxii was first isolated it was kept on a basal medium of $5 \%$ malt agar (made from malt extract $A$ without oil) on which it grew quickly and well. 
Prolonged subculture on this medium, however, led to some loss of its osmophilic properties, and thereafter $23 \%(w / w)$ glucose was added, and the cultures were stored at $4^{\circ}$ and subcultured every 3 months. Inocula for most experiments were still taken from 3-day-old cultures on the basal medium, however, as one subculture on this substrate did not affect the osmophilic properties, and growth was quicker and more prolific than with the added glucose.

Cultures and experiments were incubated at $30^{\circ}$ unless otherwise stated.

Microscopic observations on vegetative cells were made either on the living organisms or on cotton blue and lacto-phenol mounts. A satisfactory spore stain was the malachite green and safranin method (Smith, 1946) but with the omission of the alcohol wash.

Cell counts of suspensions for inoculation purposes were made on a haemocytometer.

Of several methods which were tried for the estimation of the moisture content of malt extract, a slight modification of the 'Buyze-pile' method described by Mossel \& Wit (1951) proved by far the most consistent and the easiest to use. As the only drying oven available ran at $98^{\circ}$, drying was carried on overnight instead of for 3-5 hr.

Because of the difficulty of estimating the osmotic pressure of malt extract and heavy syrups, the related property of relative humidity was used to measure the 'physiological dryness' of the various substrates. This was determined by the usual method of exposing samples to the atmospheres of saturated solutions of various salts until equilibrium is reached, the samples being preserved from mould growth by the addition of phenyl mercuric nitrate. Equilibrium was obtained in duplicate samples in 5-6 weeks. The method was also used to adjust malt extract to known relative humidities for fermentation tests.

\section{Isolation and identification of the fermenting organism}

Isolations from the original fermented samples yielded thirty-one yeasts and several bacteria, each of which was tested for its ability to ferment malt extract. After 3 weeks incubation, twenty-four of the yeasts had fermented the substrate, while the bacteria and the remainder of the yeasts had not. Of the twenty-four fermenting yeasts isolated, twenty-two were budding yeasts, very similar to one another, and referable to the subgenus Zygosaccharomyces. Five of those showing the most widely divergent characteristics on malt agar were identified at the Centraalbureau voor Schimmelcultures as Z. japonicus Saito (now transferred to $S$. rouxii Boutroux). It could thus reasonably be concluded that $S$. rouxii was the organism chiefly concerned in the fermentation.

All the experimental work now to be described was carried out with one isolate, G1, the original culture of which was subjected to three successive single-colony re-isolations. Parallel experiments were sometimes run using other isolates, and these always gave similar results. 


\section{RESULTS}

\section{Effect of temperature on growth and fermentation}

Partially sterilized malt extract was inoculated with isolate G1 at three different yeast concentrations, the precise level being measured by plate counts. The inoculated malt was filled into clear-glass jars according to the method already described, and these were incubated at $20,25,30,35$ and $37^{\circ}$. Fermentation occurred at the same time in replicates of each treatment. After 32 days, yeast counts were made on one jar from each treatment. The results are shown in Table 1.

Table 1. The effect of temperature on the fermentation of malt extract

\begin{tabular}{|c|c|c|c|}
\hline Temperature & $\begin{array}{c}\text { Initial yeast } \\
\text { count (yeasts/g.) }\end{array}$ & $\begin{array}{l}\text { Approximate time } \\
\text { for the undoubted } \\
\text { onset of } \\
\text { fermentation } \\
\text { (days) }\end{array}$ & $\begin{array}{c}\text { Yeasts/ g. after } \\
32 \text { days }\end{array}$ \\
\hline $20^{\circ}$ & $\begin{array}{r}1,515 \\
17,700 \\
462,300\end{array}$ & $\begin{array}{l}28 * \\
28 * \\
28 *\end{array}$ & $\begin{array}{r}630 \\
1,920 \\
35,800\end{array}$ \\
\hline $25^{\circ}$ & $\begin{array}{r}1,515 \\
17,700 \\
462,300\end{array}$ & $\begin{array}{r}28 * \\
<28 \\
18\end{array}$ & $\begin{array}{r}1,400 \\
97,000 \\
397,000\end{array}$ \\
\hline $30^{\circ}$ & $\begin{array}{r}1,515 \\
17,700 \\
462,300\end{array}$ & $\begin{array}{r}<28 \\
22 \\
10\end{array}$ & $\begin{array}{r}43,100 \\
332,000 \\
723,000\end{array}$ \\
\hline $\mathbf{3 5}^{\circ}$ & $\begin{array}{r}1,515 \\
17,700 \\
462,300\end{array}$ & $\begin{array}{r}28 \\
<28 \\
10\end{array}$ & $\begin{array}{l}120,000 \\
398,000 \\
318,000\end{array}$ \\
\hline $\mathbf{3 7 ^ { \circ }}$ & $\begin{array}{r}1,515 \\
17,700 \\
462,300\end{array}$ & $\begin{array}{c}\text { No fermentation } \\
<40 \dagger \\
10\end{array}$ & $\begin{array}{r}1,140 \\
27,000 \\
17,200\end{array}$ \\
\hline
\end{tabular}

* In these cases fermentation never passed beyond the scattered bubble stage.

+ Fermentation began to be apparent after the malt had been stirred for sampling (ef. p. 16).

Ingram (1949) found that in concentrated orange juice approximately $10^{6} \mathrm{cells} / \mathrm{ml}$. were necessary to cause visible fermentation. In comparison with this figure, some of those quoted for malt extract are extremely low, even taking into account that the yeast counts were made some time after the actual start of the fermentation, by which time the number of cells would be expected to be decreasing. A probable explanation lies in the physical consistency of malt extract, which is so thick that it would hold locally small bubbles of gas until enough accumulated to make a large visible bubble. In view of this, it is possible that those samples of extract in Table 1, which never reached the frothing stage, should not be considered to have fermented, in the ordinary sense of the word, even though there were demonstrably more bubbles present than in uninoculated extract. 
Another possible cause for low yeast counts in the fermented extract is the plate-count method used to determine them, which was made necessary by the nature of the substrate. Though microscopic observation of the sediment from diluted centrifuged malt extract did not indicate clumping of cells to any extent, yet any clumping would of necessity reduce the apparent yeast count. The method, also, would fail to include fermenting but non-viable cells in the count.

Table 1 shows that the optimum temperature for fermentation lies between 30 and $35^{\circ}$; but it also shows obvious peculiarities in the behaviour of the yeast at $37^{\circ}$, for though fermentation is as quick as at any temperature at the highest yeast concentration, it fails to take place at all at the lowest. Malt extract darkens considerably when kept at $37^{\circ}$ for 3 or 4 days, but no gas bubbles have been noticed during periods of storage lasting up to 3 months. The type of browning appears, therefore, to be of a different character from that of dried fruits and concentrated fruit juices stored at high temperatures, for in these products it is usually accompanied by the evolution of $\mathrm{CO}_{2}$ (Stadtman, 1948). The darkened malt extract was not in itself toxic to $S$. rouxii, as in samples inoculated with the yeast and incubated at $30^{\circ}$ the organism multiplied in the usual way.

For a more detailed study of the fate of the organism at $37^{\circ}$ malt extract was inoculated with isolate G1 to give a count of approximately 12,000 yeasts/g. and was filled into glass jars and stored at this temperature. One jar was removed from storage each week for a number of weeks, and a yeast count made, the results being given in Table 2. It is clear that $S$. rouxii

\section{Table 2. The effect of storage at $37^{\circ}$ on the yeast} count of malt extract

$\begin{array}{cc}\begin{array}{c}\text { Storage time } \\ \text { (weeks) }\end{array} & \text { Yeasts/g. } \\ 0 & 12,000 \\ 1 & 10,150 \\ 2 & 36,800 \\ 3 & 47,600 \\ 4^{*} & 66,300 \\ 5 & 7,010 \\ 6 & 200 \\ 7 & 0 \\ 8 & 0\end{array}$

* Fermentation was visible by this time in all jars.

cannot tolerate prolonged storage at $37^{\circ}$, though it can continue to multiply for 4 weeks. The results shown in Table 1 can thus be explained if we consider that a small inoculum cannot multiply sufficiently in these 4 weeks to cause visible fermentation, while a larger inoculum can multiply and/or metabolize sufficiently to do so before it dies. That the sudden dying-off of the yeast after 4 weeks is not due to any toxic byproducts of fermentation is shown by the fact that the cells can survive in fermented extract at $30^{\circ}$ for many months.

A microscopic examination of infected malt extract which had been stored 
at various temperatures showed that sporing, frequently with conjugation, occurred over the whole temperature range between 4 and $37^{\circ}$, but that active growth was most conspicuous at $30^{\circ}$.

\section{Effect of composition of the substrate on growth and fermentation}

The property of a highly concentrated substrate most likely to affect critically the growth and survival of any yeast colonizing it, would be its osmotic pressure, a factor dependent both on the water content and on the relative proportion of osmotically active to osmotically inactive substances. These properties of malt extract were therefore investigated in relation to the growth of $S$. rouxii, using relative humidity as a measure of the osmotic strength of the substrate, for reasons already stated.

Heavy inoculation of samples from different batches of mixtures $\mathbf{A}$ and $\mathbf{B}$, spread over their natural range of moisture contents, caused all samples of mixture $A$ to ferment within 46 days, while mixture $B$ not only failed to ferment in 2 months, but very few live yeasts were found in it at the end of this time.

The effect of adjusting the moisture contents of the two malts was then investigated. That of a sample of mixture $A$ was reduced to $17 \%$ by exposing it to the atmosphere of a saturated solution of potassium iodide until its weight remained constant, when an inoculum sufficiently heavy to ferment normal mixture $A$ in a few days was added. No fermentation was apparent after 5 weeks, though the yeast was still alive at this time. In the case of mixture $B$, the addition of water rather than its removal was necessary. Extract adjusted to contain $22.9 \%$ moisture began to ferment in 26 days, but that adjusted to $\mathbf{2 2 . 2} \%$ moisture failed to ferment in 12 weeks. It is thus clear that, while moisture content can act as the limiting factor on the growth of $S$. rouxii in malt extract, there is no absolute value, constant from extract to extract. Some other property of malt, partly dependent on the moisture content, must be looked for.

The equilibrium relative humidities of samples of both extracts were therefore determined by the method already mentioned, and the curves obtained are shown in Fig. 1. The initial moisture contents of the samples of mixtures $A$ and $B$ used for this experiment were 20.9 and $20.6 \%$ respectively, the equilibrium relative humidities as read from the graph are therefore $75 \cdot 4$ and $71.4 \%$, a difference of $4 \%$. The approximate limiting moisture contents for fermentation, as already determined, lie, for both extracts, at about the same value of relative humidity, $73.3 \%$. So that it is now possible to say (subject to the fact that only two extracts have been studied), that total osmotic pressure, expressed here as relative humidity, is the controlling factor determining whether a given sample of malt extract can be fermented by S. rouxii.

Now, it has already been pointed out that the total osmotic pressure of malt extract depends both on the moisture content and on the combined osmotic strength of the soluble constituents. But experiment has shown that extracts $A$ and $B$ have very similar moisture contents, so that this cannot account for 
the difference in their relative humidities, and hence in their fermentability, which must, therefore, lie in the total osmotic pressures of the soluble constituents. As it proved difficult to obtain an accurate analysis of the solids of the two malt extracts, and so to compare them directly, it was decided instead to measure and compare their total osmotic strengths through the property of depression of the freezing-point of water, and Dr R. E. Stuckey, of the

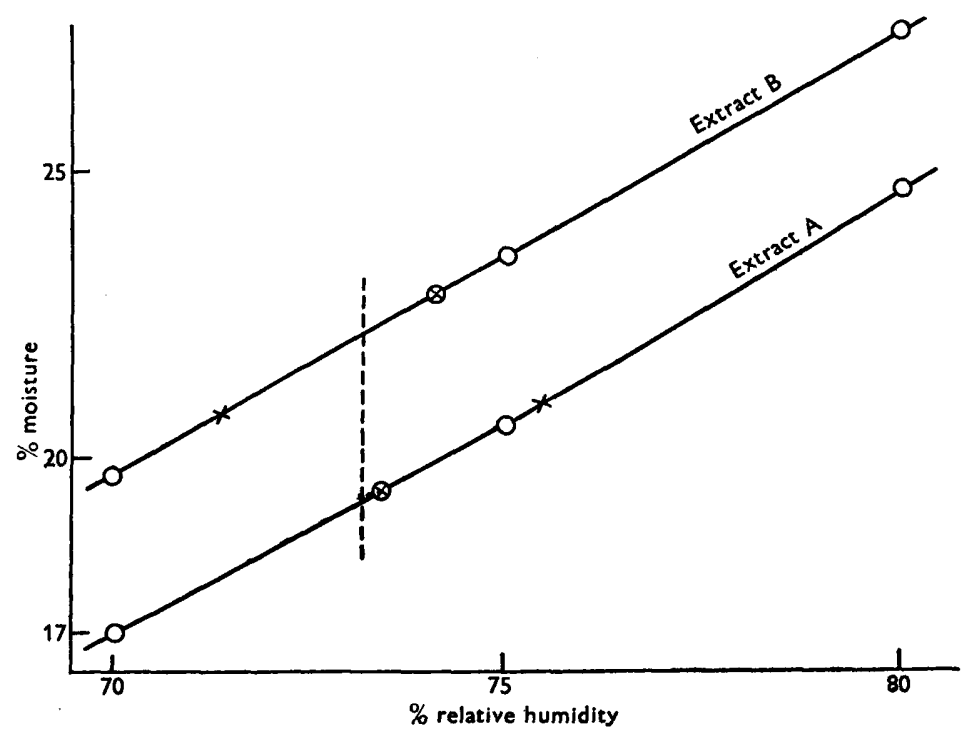

Fig. 1. Moisture content and relative humidity of malt extract mixtures $A$ and $B$. $\otimes$, lowest moisture content at which mixture is known to ferment; $x$, relative humidity of the untouched mixture. The broken line indicates approximately the relative humidity below which neither mixture can be fermented by $S$. rouxii.

Analytical Department, kindly undertook this estimation. The results, expressed in terms of the equivalent percentage $(w / w)$ of glucose, were as follows:

$\begin{array}{ccc}\text { Mepression of } & \begin{array}{c}\text { Dep } \\ \text { freezing point }\end{array} & \begin{array}{c}\text { Equivalent } \% \\ \text { glucose }\end{array} \\ \text { A } & \left({ }^{\circ}\right) & \mathbf{3 9 \cdot 9} \\ \text { B } & 1 \cdot 165 & 47 \cdot 6\end{array}$

thus confirming the view that it is the difference in osmotic strength of the ingredients that is the cause of the difference in fermentability of the two mixtures.

The general conclusion can now be drawn, that $S$. rouxii cannot ferment a malt extract with a relative humidity of $78 \%$ or less, and that the relative humidity can be adjusted either by altering the moisture content or the proportion of sugars to non-sugars in the solids content. 


\section{DISCUSSION}

Fabian \& Hall (1933) attempted to find the exact level of moisture content at which artificially inoculated honey would begin to ferment, by standing the infected honey in a saturated atmosphere and determining the moisture content when the first visible signs of fermentation appeared. But we know (Kroemer \& Krumbholz, 1931; von Schelhorn, 1950) that visible fermentation in highly concentrated media takes a long time to set in, probably (Ingram, 1949) due to slow multiplication of the yeast; so that with a continually increasing moisture content, as in Fabian \& Hall's experiment, fermentation could well have taken place at moisture levels below those actually recorded if the honey had remained long enough at these levels. In fact, after describing this experiment, in which the most concentrated honey used contained $32.8 \%$ moisture at first, but did not ferment visibly until it contained $34 \cdot 1 \%$, Fabian \& Hall mention that a desiccated honey containing 'about $10 \%$ ' of moisture was slowly fermented when inoculated with their 'best fermenting yeast'. It seems, therefore, that in this experiment the authors are measuring the time taken for visible fermentation to set in, rather than the lowest moisture level at which it is possible.

If $S$. rouxii is compared with other xerophilic and osmophilic organisms for which figures are available, it is seen to be somewhat less resistant than many of them. Stille (1948) found, for instance, that Aspergillus glaucus could produce spores at a relative humidity of $70 \%$, a level which kills $S$. rouxii in 2 months, while von Schelhorn (1950) has shown the extraordinary resistance of selected strains of $\mathbf{Z}$. barkeri which can grow slowly in fructose syrups of $62 \%$ relative humidity. It is possible that with careful selection and 'training' a more resistant strain of $S$. rouxii could be obtained. Though Fabian \& Quinet (1928), Fabian \& Hall (1933) and Lochhead (1933) gave limits of moisture content below which naturally infected honey and maple syrup would not ferment, they did not give the relative humidities of the substrates, making it difficult to compare their results with others. The results described in this paper agree with those of other investigators (see Mossel \& Westerdijk, 1949) in demonstrating that in systems of high sugar (or salt) content the controlling factor for the growth of yeasts is not the absolute moisture content, but the availability of the moisture present as measured by the osmotic pressure or relative humidity of the substrate. They show more clearly than most, however, the exact relationship between the sugar and moisture content of the system in their effect on the final relative humidity. The recent work of Schachinger (1950) bears directly on this point. He shows that for bakers' yeast growth ceases completely in $\mathbf{4 7} \%$ glucose or fructose (monosaccharide) solutions, but in sucrose (disaccharide) syrup, it can continue up to a concentration of $60 \%$. All these solutions have a relative humidity of about $90 \%$. For osmophilic yeasts the same effect was noted, but the limiting sugar concentrations were considerably higher.

In the case of malt extract, for equal moisture contents, the samples most likely to ferment would be those that had undergone the least hydrolysis 
during the manufacture of the malt, and hence would have comparatively high dextrin/maltose and maltose/glucose ratios and correspondingly high relative humidities. Samples which had undergone more complete hydrolysis and contained comparatively high proportions of sugars, especially glucose, would be those likely to inhibit yeast growth. An examination of different malt extracts with this observation in mind would be of interest.

My thanks are due to Dr Kreger van Rij of the Centraalbureau voor Schimmelcultures, who identified the original yeast isolates for me, and Miss Sloof, who advised on their nomenclature : to Dr C. J. Hickman of the University of Birmingham, for his criticism of the manuscript, and to Dr C. Rainbow of the same University, who also read the manuscript: to Dr S. W. F. Underhill of this Department for his continued encouragement; and to the Directors of The British Drug Houses Ltd. for permission to publish these results. Most particularly, I should like to thank Mr J. T. Gunner and Miss Constance Rainbow for their technical assistance.

\section{REFERENCES}

Boutroux, M. L. (1884). Sur la conservation des ferments alcooliques dans la nature. Ann. Sci. nat. (Bot.), 6, 144.

English, M. P. (1951). Fermentation of malt extract. Nature, Lond. 168, 391.

Fabian, F. W. \& Hali, H. H. (1933). Yeasts found in fermented maple syrup. Zbl. Bakt. (Abt. 2), 89, 31.

Fabian, F. W. \& Quinet, R. I. (1928). A study on the cause of honey fermentation. Tech. Bull. Mich. agric. Exp. Sta. 48, no. 92.

Ingram, M. (1949). Fermentation in concentrated orange juice. Food Manuf. 24, 77.

Joslyn, M. A. \& Creuss, W. V. (1929). A comparative investigation of certain film-forming fungi. Hilgardia, 4, 201.

Kroemer, K. \& Krumbiolz, G. (1931). Untersuchungen über osmophile Sprosspilze. I. Arch. Mikrobiol. 2, 352.

Lochread, A. G. (1983). Factors concerned with the fermentation of honey. $Z$ Zbl. Bakt. (Abt. 2), 88, 296.

Lodder, J. \& Kreger van RiJ, N. J. W. (1952). The Yeasts, a Taxonomic Study. Amsterdam: Noord-Hollandsche Uitgeversmij.

Mossel, D. A. A. (1951). Investigation of a case of fermentation in fruit products, rich in sugars. Leeurvenhoek ned. Tijdschr. 17, 146.

Mossei, D. A. A. \& WesterdiJk, J. (1949). The physiology of microbial spoilage in foods. Leeurwenhoek ned. Tijdschr. 15, 190.

Mossex, D. A. A. \& WiT, S. L. (1951). Determination of the water content of foods. III. Analyt. Chim. acta, 5, 63.

Mrak, E. M. \& Phaff, H. J. (1948). Yeasts. Annu. Rev. Microbiol. 2, 1.

Mrak, E. M., Phaff, H. J. \& Vaughn, R. H. (1942). Yeasts occurring on dates. J. Bact. 43, 689.

SaIto, K. (1907). Mikrobiologische Studien über die Soyabereitung. Zbl. Bakt. (Abt. 2), 17, 20.

Schachinger, L. (1950). Osmotischer Druck und Mikroorganismenwachstum auf Lebensmitteln. Z. LebensmittUntersuch. 91, 423.

SchelHorN, M. von (1950). Untersuchungen über den Verderb wasserarmer Lebensmittel durch osmophile Mikroorganismen. I. Z. LebensmittUntersuch. 91, 117 .

Schelhorn, M. von (1951). Control of micro-organisms causing spoilage in fruit and vegetable products. Advanc. Food Res. 3, 429.

Sмттн, G. (1946). An Introduction to Industrial Mycology. London: Arnold, p. 56. 
Stadtman, E. R. (1948). Nonenzymatic browning in fruit products. Advanc. Food Res. $1,325$.

StiLLe, B. (1948). Grenzwerte der relativen Feuchtigkeit und des Wassergehaltes getrockener Lebensmittel für den mikrobiellen Befall, Z. LebensmittUntersuch. 88, 9.

Whaluey, H. C. S. de \& Scarr, M. P. (1947). Micro-organisms in raw and refined sugar and intermediate products. Chem. \& Industr. 66, 531.

\section{Note on a Method of Vital Staining for Saccharomyces rouxii}

By J. T. Gunner and Constance Rainbow

A number of dyes were investigated as possible vital stains for $S$. rouxii, and of these Trypan Blue proved the most satisfactory. After some trial, the following method was found suitable for the routine counting of yeast colonies in dilution plates.

A $0 \cdot 1 \%$ solution of Trypan Blue in sterile distilled water was boiled for 5 min. and kept aseptically as stock. When required, enough of this solution was added to the tubed, melted agar medium to give a final concentration of 1/10,000 Trypan Blue, and plates were poured.

The stain at this concentration was entirely nontoxic to $S$. rouxii, which absorbed it readily to give very dark blue colonies easily visible against the light blue of the agar.

(Received 31 October 1952) 